\title{
Movement of magnetic domain walls induced by single femtosecond laser pulses
}

\author{
O. Sandig, ${ }^{1}$ Y. A. Shokr, ${ }^{1}$ J. Vogel,,${ }^{2,3}$ S. Valencia,${ }^{4}$ F. Kronast,${ }^{4}$ and W. Kuch ${ }^{1, *}$ \\ ${ }^{1}$ Institut für Experimentalphysik, Freie Universität Berlin, Arnimallee 14, 14195 Berlin, Germany \\ ${ }^{2}$ CNRS, Institut Néel, 38042 Grenoble, France \\ ${ }^{3}$ Université Grenoble Alpes, Institut Néel, 38042 Grenoble, France \\ ${ }^{4}$ Helmholtz-Zentrum Berlin für Materialien und Energie, Albert-Einstein-Straße 25, 12489 Berlin, Germany
}

(Received 3 May 2016; revised manuscript received 8 July 2016; published 11 August 2016)

\begin{abstract}
We present a microscopic investigation of how the magnetic domain structure in ultrathin films changes after direct excitation by single ultrashort laser pulses. Using photoelectron emission microscopy in combination with $\mathrm{x}$-ray magnetic circular dichroism in the resonant absorption of soft $\mathrm{x}$ rays, we find that individual laser pulses of $\approx 60 \mathrm{fs}$ duration and a central wavelength of $800 \mathrm{~nm}$ lead to clear changes in the domain structure of a Co layer of three atomic monolayers thickness in an epitaxial $\mathrm{Co} / \mathrm{Cu} / \mathrm{Ni}$ trilayer on a $\mathrm{Cu}(001)$ single-crystal substrate. A relatively small enhancement of the sample base temperature by $40 \mathrm{~K}$ is sufficient to lower the threshold of laser fluence for domain wall motion by about a factor of two. Pump-probe measurements with a laser fluence just below this threshold indicate that the laser-induced demagnetization of the sample is far from complete in these experiments. Although the domain wall motion appears similar to thermal domain wall fluctuations, quantitatively it cannot be explained by pure thermal activation of domain wall motion by the transient rise of sample temperature after the laser pulse, but it is likely to be triggered by a laser-induced depinning of domain walls.
\end{abstract}

DOI: 10.1103/PhysRevB.94.054414

\section{INTRODUCTION}

The fast manipulation of magnetization without resorting to magnetic fields or electric currents is one of the key issues for the further development of magnetic memory storage or information processing. Of particular interest is the possibility to use ultrashort laser pulses for the local manipulation or reversal of magnetization. Heat-assisted magnetic recording, for example, takes advantage of local heating of the magnetic storage media by a focused laser pulse, which reduces the magnetic anisotropy barrier for field-driven magnetization reversal [1,2]. All-optical magnetization control, i.e., without the help of any magnetic or electric fields, promises the highest speed for writing of magnetic information and is thus highly attractive for applications. Apart from laser-pulse-induced ultrafast demagnetization [3-5], laser pulses can also reverse the magnetization. Circularly polarized laser pulses can act on the magnetization via the inverse Faraday effect [6], and all-optical helicity-dependent switching has been observed in a range of materials [7]. In these experiments, the laser pulse drives the material close to the paramagnetic phase transition while the final magnetization state is then determined by the helicity of the driving laser pulse. In ferrimagnetic materials, on the other hand, a toggle switching of the magnetization has been observed, independent of the light polarization [8]. Due to an intensity threshold for switching in connection with the helicity dependence of the absorption in magnetic materials (magnetic circular dichroism), this also leads to helicity-dependent all-optical switching [9].

A laterally inhomogeneous response after ultrashort laser excitation can result from the flow of angular momentum by photoexcited spin-polarized electrons [10-14]. This can lead to, for example, locally different demagnetization times

*kuch@ physik.fu-berlin.de in inhomogeneous magnetic alloys [15] or a transient spatial widening of magnetic domain walls [16]. An optically induced lateral macroscopic displacement of domain walls by individual laser pulses has not been reported so far, although controlling domain wall displacement is important for applications such as racetrack memories [17]. A reason may be that domain wall velocities are typically no more than $10^{3} \mathrm{~m} / \mathrm{s}$ [18], such that the timescale for domain wall motion is orders of magnitude larger than that of the exciting ultrashort laser pulse. Any domain wall motion induced by ultrashort laser pulses must thus either rely on material properties the change of which persists after the end of the actual excitation such as the temperature increase [19,20], or on inertia of the magnetic domain wall motion [21-24].

Here, we demonstrate that single laser pulses of $\approx 60 \mathrm{fs}$ duration can lead to a local switching of magnetization by moving domain walls without applied magnetic field. Using photoelectron emission microscopy (PEEM) with $\mathrm{x}$-ray magnetic circular dichroism (XMCD) as the magnetic contrast mechanism we observe stochastic movements of magnetic domain walls in a $\mathrm{Co} / \mathrm{Cu} / \mathrm{Ni}$ trilayer triggered by excitation with individual infrared laser pulses. The intensity of these pulses is clearly below the one necessary for complete demagnetization of the sample, as evidenced by time-resolved pump-probe experiments. The distance traveled by an individual domain wall after one pulse - up to several hundred nanometers-is much longer than what a domain wall could possibly move within the duration of the laser pulse without assuming unrealistically high domain wall velocities. It implies that the domain wall motion must persist for some time after the laser pulse. Although the pattern of domain wall motion resembles that of thermal domain fluctuations [25-27], the temperature dependence of the probability clearly shows that thermally induced domain wall motion due to the transient heating by the laser pulse alone is not able to explain the effect. We suggest a two-step mechanism based on laser-pulse-induced depinning 
of domain walls and successive thermal domain wall motion after the laser pulse as the underlying process.

\section{EXPERIMENT}

The experiments were performed at beamline UE49SPEEM of the synchrotron radiation facility BESSY II (Helmholtz-Zentrum Berlin) in Berlin, Germany. The sample was prepared in an ultrahigh vacuum preparation chamber connected to the PEEM end station. The base pressure was $2 \times 10^{-9}$ mbar. During the evaporation process, the pressure did not exceed $3 \times 10^{-9}$ mbar. The $\mathrm{Cu}(001)$ crystal was cleaned by $\mathrm{Ar}^{+}$bombardment, first with energy of $1.0 \mathrm{keV}$, followed by softer sputtering with an energy of $0.5 \mathrm{keV}$. The sample was then annealed to a temperature of $820 \mathrm{~K}$ for $10 \mathrm{~min}$. The trilayer system three atomic monolayers (ML) $\mathrm{Co} / \mathrm{Cu} / 15 \mathrm{ML} \mathrm{Ni}$ was grown by thermal evaporation onto the $\mathrm{Cu}(001)$ substrate held at room temperature. Ni and Co were evaporated by electron bombardment of rods of high-purity $(99.9 \%)$ material while $\mathrm{Cu}(99.9 \%)$ was heated by electron bombardment of a molybdenum crucible.

At these thicknesses, cobalt shows in-plane magnetization and nickel out-of-plane magnetization [28]. The $\mathrm{Cu}$ layer was grown as a wedge, ranging from 0 to $6 \mathrm{ML}$ in thickness within $100 \mu \mathrm{m}$ by placing a knife edge in front of the sample. This allows us to select a certain $\mathrm{Cu}$ thickness and hence a certain interlayer coupling strength in the PEEM experiments by choosing a certain position on the sample.

The sample was imaged with an Elmitec PEEM-II instrument [29] using low-energy electrons emitted from the sample surface after absorption of $\mathrm{x}$ rays $[30,31]$. To enhance the spatial resolution, energy filtering of the electrons with about $1 \mathrm{eV}$ resolution was applied. The acceleration potential between sample and first objective lens of the PEEM was $10 \mathrm{keV}$. The $\mathrm{x}$ rays and the laser illuminate the sample surface from opposite sides at a grazing incidence angle of $16^{\circ}$. Overlap of the laser spot with the $\mathrm{x}$ rays can be confirmed by imaging the laser-excited photoelectrons at the sample surface. Because of the low photon energy of the laser of $1.55 \mathrm{eV}$, these photoelectrons are generated by nonlinear processes, mainly three-photon photoemission. This photoemission is dominated by hot spots at the sample surface such as topographic defects [32] but can be used to locate the laser spot on the surface. The $\mathrm{X}$ rays have a spot size $(\mathrm{FWHM})$ of about $20 \times 30 \mu \mathrm{m}^{2}$ and a duration of about 50 ps. The laser used was a Femtosource Scientific XL Ti sapphire oscillator with a repetition rate of $5 \mathrm{MHz}$, synchronized to the revolution frequency of $1.25 \mathrm{MHz}$ of the electron storage ring of the synchrotron light source. The maximum energy per pulse was $300 \mathrm{~nJ}$. Each pulse had a duration of about $60 \mathrm{fs}$ with a central wavelength of $800 \mathrm{~nm}$. To perform single-shot experiments, the laser system was combined with a Femtolasers Pulsfinder. With this setup it is possible to select any repetition rate ranging from $5 \mathrm{MHz}$ down to a single shot. The laser was focused by an optical lens inside the vacuum chamber to a spot size of $10 \times 30 \mu \mathrm{m}^{2}$ on the sample (at $1 / e$ ). To vary the flux density, a combination of a $\lambda / 2$ plate and a polarizer was used. This allowed us to tune the fluence, which in the center of the spot ranged from 0 to $110 \mathrm{~mJ} \mathrm{~cm}^{-2}$. The numbers specified in the following always refer to the fluence averaged over one standard deviation of the elliptic Gaussian beam profile and include a systematic error of about $25 \%$. At the sample, the laser pulses are linearly $p$ polarized with a degree of polarization of more than $95 \%$.

Pump-probe experiments have been performed at a sample temperature of $293 \mathrm{~K}$, using radiation from the single bunch in the BESSY II hybrid mode of operation, which is separated from the train of other pulses by 100 ns. To blank out the electrons excited by the remaining x-ray pulses, the imaging unit of the PEEM was gated by voltage pulses, which reduce the sensitivity to all other x-ray pulses except the single pulse such that the other pulses lead to a background of only about $5 \%$ of the total intensity. Laser pulses of $1.25 \mathrm{MHz}$ frequency are obtained by operating the pulse picker with 1:4 ratio. The delay time between $\mathrm{x}$-ray and laser pulse is varied continuously by a trombone inserted into the synchronization line and, in addition, electronically in steps of $2 \mathrm{~ns}$.

Magnetic domain images presented in the following have been acquired with a fixed helicity of the circularly polarized $\mathrm{x}$ rays and a photon energy tuned to the maximum of the Co $L_{3}$ absorption peak. Except for the small stray field of the objective lens, no external magnetic fields are present during the measurements. Since the X-ray spot on the sample is an image of the exit slit of the monochromator, there is a photon energy dispersion of about $1 \mathrm{eV}$ across a field of view of $15 \mu \mathrm{m}$. In the selected region, the $\mathrm{Cu}$ thickness was such that the Ni magnetization displays a clear tilt in the direction of the Co magnetization, without being pulled completely into the in-plane direction. This corresponds to a $\mathrm{Cu}$ thickness of about 3.5-4.0 ML [28].

\section{RESULTS AND DISCUSSION}

Figure 1(a) shows a typical PEEM image of the magnetic domains in the Co layer. The field of view is $15 \mu \mathrm{m}$. The grayscale contrast represents the local $\mathrm{x}$-ray absorption at the maximum of the Co $L_{3}$ edge for positive helicity of the x rays. Due to XMCD, brighter (darker) areas correspond to a more parallel (antiparallel) projection of the local magnetization direction on the direction of the incoming $\mathrm{x}$ rays, indicated by the arrow in Fig. 1(a). Brighter and darker gray areas in Figs. 1(a) and 1(b) correspond to domains with opposite magnetization direction. The red ellipse indicates roughly the footprint of the laser spot, which can be recognized from three-photon photoemission when enhancing the sensitivity of the image converter while blocking the $\mathrm{x}$ rays.

To see the effect of individual laser pulses, a series of images with a fixed x-ray helicity was acquired. After acquisition of each image, which took $10 \mathrm{~s}$, one single laser pulse was applied to the sample, then the next image was acquired, and so on. The images presented in Fig. 1(b) are part of such a series. They show the region marked by the blue square in Fig. 1(a). Between each image, one laser pulse with a fluence of $F=14.4 \mathrm{~mJ} \mathrm{~cm}^{-2}$ was applied. Overall, the main domain configuration remains similar. Locally, however, some domains change as a consequence of the laser pulse. These differences can be seen more conveniently in Fig. 1(c), which shows images calculated as the pixel-by-pixel forward difference of two consecutive Co domain images of Fig. 1(b). Dark and white areas here represent places in which the magnetization direction has reversed, either from more parallel 


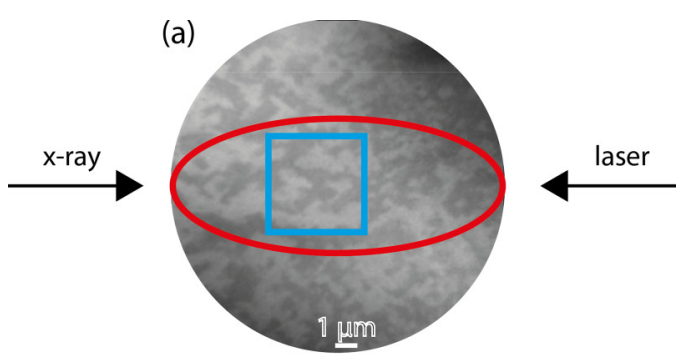

(b)
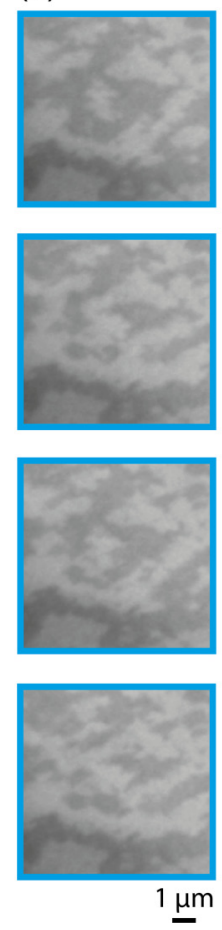

(c)
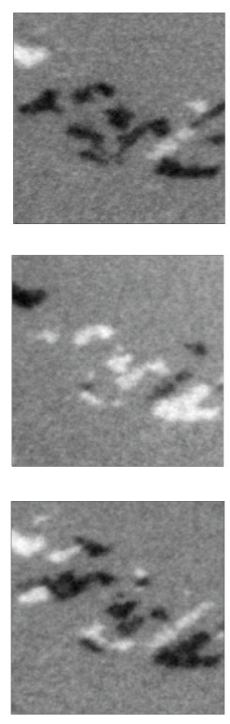

$1 \underline{\mu m}$

FIG. 1. (a) PEEM domain image at the Co $L_{3}$ edge, where brighter and darker gray areas correspond to domains with opposite magnetization direction. The ellipse indicates the footprint of the laser spot. The square marks the magnified region which is shown in the images of (b). (b) Image series showing the effect of single laser pulses with a fluence of $F=14.4 \mathrm{~mJ} \mathrm{~cm}^{-2}$ on the magnetic domain structure. Between two consecutive images one laser pulse is applied. (c) The forward difference of two consecutive images of (b). Dark and white contrast indicates areas in which the local magnetization direction has changed by the laser pulse, while intermediate gray contrast indicates areas with no effect. Sample temperature $\approx 325 \mathrm{~K}$.

to more antiparallel to the direction of the incoming $\mathrm{x}$ rays (bright) or vice versa (dark). Areas which are not affected by the laser pulse remain identical in the domain images and appear thus with an intermediate gray contrast in the difference images. Closer inspection of these images reveals that the shape and size of individual domains changes only little. This is a hint that the fluence of the laser pulses is not sufficient to fully demagnetize the sample.

Pump-probe experiments have been performed in order to further confirm this. The result of such measurements, performed on a similar wedge at identical film thicknesses, is condensed into Fig. 2. When the laser fluence is high enough to yield changes to the domain structure after single laser pulses like the ones shown in Fig. 1, the XMCD domain (a)

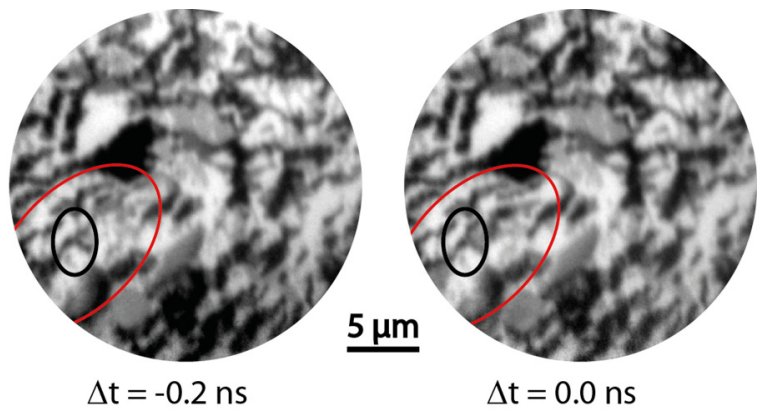

(b)

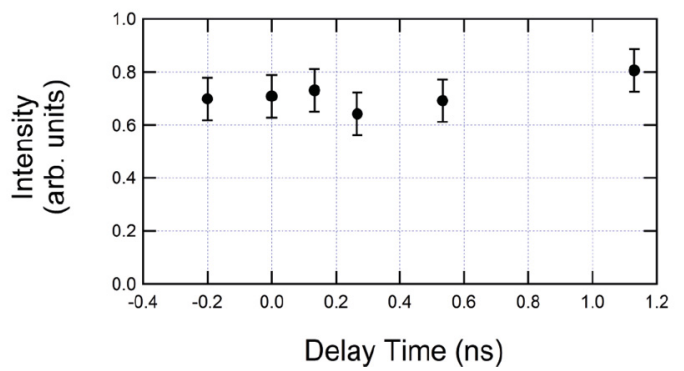

FIG. 2. (a) Pump-probe XMCD domain images at the Co $L_{3}$ edge for two delay times between pump and probe beam, left: $-0.2 \mathrm{~ns}$, right: $0.0 \mathrm{~ns}$. The red line marks the footprint of the laser pulse. (b) Pump-probe XMCD contrast between Co domains within the area marked by the black line as a function of delay time between pump and probe pulses. The negligible variation around zero time delay proves that the sample is not demagnetized by the laser pulse. The laser fluence is $F=13.1 \mathrm{~mJ} \mathrm{~cm}^{-2}$. Error bars have been determined from the variation of the XMCD contrast outside the laser-illuminated region.

contrast in pump-probe images disappears in the region where the laser beam hits the sample due to nonrepetitive changes to the domain structure after each laser pulse. Because only reproducible changes to the domain structure can be seen in pump-probe experiments, the fluence has to be lower than the threshold above which domain changes are observed. Reducing the laser fluence just below that threshold, pumpprobe domain images taken before, at, and after zero time delay between laser pump and x-ray probe show basically no difference in the XMCD domain contrast. Figure 2(a) shows two time-resolved Co XMCD images recorded at $\Delta t=-0.2 \mathrm{~ns}$ and $\Delta t=0.0 \mathrm{~ns}$ for a laser fluence of $13.1 \mathrm{~mJ} \mathrm{~cm}^{-2}$. Black and white correspond to domains with opposite magnetization, while gray contrast indicates magnetization rotated by $90^{\circ}$. The magnetic contrast in both images is similar. Figure 2(b) shows an analysis of the XMCD domain contrast as a function of delay time between laser and x-ray pulses. There is no significant decrease of the domain contrast around zero time delay. Even considering the time averaging of about $50 \mathrm{ps}$ due to the pulse width of the x-ray pulse, which would broaden ultrafast changes in the XMCD signal, a complete demagnetization of the sample can be ruled out. The time constant for recovery of the magnetization after laser-pulse excitation increases with increasing sample demagnetization [33-35], reaching values of the order of 50 ps already for $40 \%$ demagnetization in Ni films [36]. A complete demagnetization of the Co layer in our samples would thus be clearly seen in our 
pump-probe experiments. We conclude that also at the slightly higher fluences, where the laser-induced domain wall motion effect is observed, the sample is most likely not completely demagnetized by the laser pulses.

In order to quantify the amount of domain changes induced by each laser pulse, a series of images of $10 \mathrm{~s}$ acquisition time interleaved with single laser pulses were acquired for increasing laser fluences, and a statistical analysis of the amount of changed area was performed. The latter was determined from discretized difference images, in which cluster size analysis was used to exclude regions with a surface smaller than 20 image pixels of $30 \times 30 \mathrm{~nm}^{2}$ in size as noise.

Figure 3 shows the Co domain pattern of the same part of the sample for three different fluences $(8.0,12.7$, and $20.0 \mathrm{~mJ} \mathrm{~cm}^{-2}$ ), increasing from top to bottom. The rainbow color code used for the superimposed contour plots indicates the local average number of switching events per pulse after approximately 30 laser pulses. Red color indicates less switching events than blue color; uncolored regions did not exhibit any switched magnetization. With increasing laser fluence the number of overall switching events is increasing. Additionally, the overall area where switching occurs increases. Nevertheless, the main magnetic domain configuration remains preserved, as can be seen by comparing the magnetic patterns in the background. Even for the highest fluence of $20.0 \mathrm{~mJ} \mathrm{~cm}^{-2}$, there are still regions well within the laser spot that do not exhibit any change of their magnetization. Both indicate that the laser pulses are indeed not fully demagnetizing the sample.

The size of the regions in which the magnetization direction has changed after single laser pulses is in the micrometer range. Their often elongated shape following the alignment and general shape of the magnetic domain pattern suggests domain wall displacements as the underlying mechanism. Viewing a series of images obtained after single laser pulses as a movie [37] reminds one of stochastic thermal domain fluctuations $[26,38]$. This supports the assumption of domain wall displacement as the responsible mechanism. It is consistent with the observation that the average width of the switched regions, which in this case would correspond to the mean domain wall displacement, increases for stronger laser pulses. To estimate these values, the switched areas in the difference images were fitted to ellipses, the minor axes of which describe the displacements of the domain walls, as shown in Fig. 4. Areas with a surface smaller than ten image pixels were excluded as noise. For the laser fluences of Fig. 3, the average displacement per pulse obtained in this way is approximately $95 \mathrm{~nm}$ for $8.0 \mathrm{~mJ} \mathrm{~cm}{ }^{-2}$ and $130 \mathrm{~nm}$ for $20.0 \mathrm{~mJ} \mathrm{~cm}^{-2}$ at a sample base temperature of $333 \mathrm{~K}$. At $293 \mathrm{~K}$, the average displacement is around $100 \mathrm{~nm}$ with no clear dependence on the laser fluence at laser fluences above threshold for domain wall motion [37]. Fitting the switched areas to rectangles leads to similar results.

A quantitative analysis of the switching probability as a function of laser fluence is presented in Fig. 5 for two different base temperatures of the sample. The switching probability is defined as the average total area of reversed magnetization after a single laser pulse and was determined from a series of domain images like the three examples presented in Fig. 3. The switching probability rises with the laser fluence above a certain threshold fluence. Below, no domain wall motion is

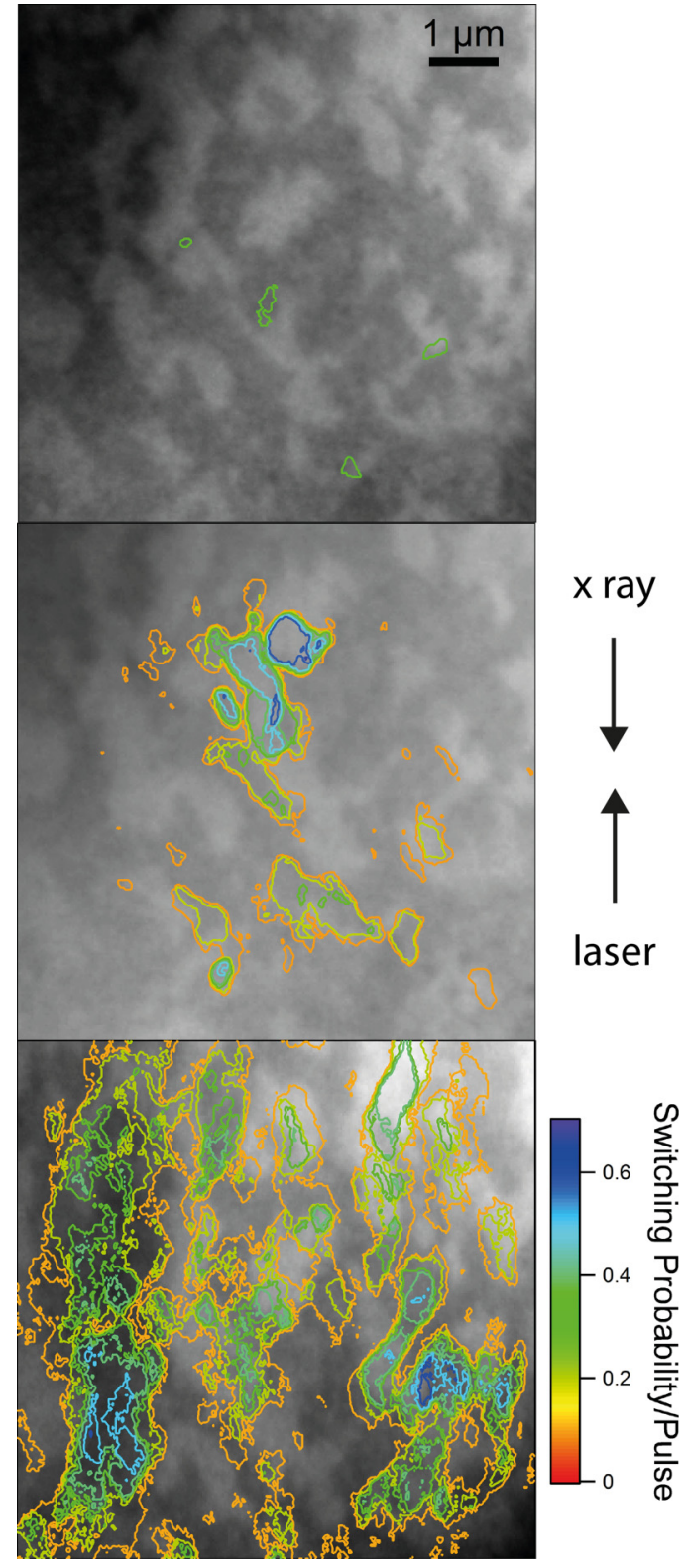

FIG. 3. PEEM domain images recorded at the Co $L_{3}$ edge with a superimposed contour plot showing the local amount of domain changes for increasing laser fluence in an image series taken at the same position. From up to down: 8.0, 12.7, and $20.0 \mathrm{~mJ} \mathrm{~cm}^{-2}$, sample base temperature: $333 \mathrm{~K}$. The grayscale contrast is identical to Fig. 1(a) and 1(b). Brighter and darker gray areas are domains with opposite magnetization direction, where brighter (darker) contrast corresponds to a more positive (negative) projection of the local magnetization direction on the direction of the incoming $\mathrm{x}$ rays, indicated by an arrow.

observed, and the domain pattern is stable over several tens of minutes without any change within the field of view both at 293 and $333 \mathrm{~K}$ sample temperature.

Interesting is the drastic change of switching probability with the sample base temperature. The moderate change of only $40 \mathrm{~K}$ leads to an about threefold increase in the switching probability at constant fluence. Much smaller laser fluences are sufficient to trigger domain wall movements at $333 \mathrm{~K}$ 


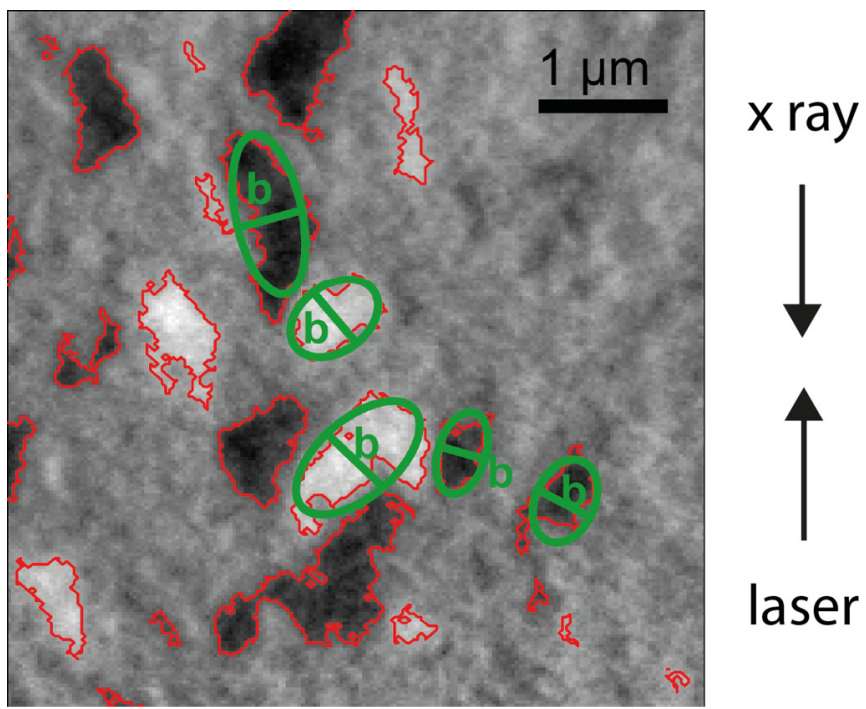

FIG. 4. Estimate of domain wall displacement. Shown is a difference image between two Co domain images taken before and after a single laser pulse of $20.0 \mathrm{~mJ} \mathrm{~cm}^{-2}$ at a sample base temperature of $333 \mathrm{~K}$. The red lines encompass regions in which the magnetization direction has reversed, where white and dark corresponds to a reversal from more parallel to more antiparallel with respect to the direction of the incoming $\mathrm{x}$ rays and vice versa, respectively. To estimate the distance of a DW movement, the marked areas were fitted with ellipses. The minor axes $b$ of these ellipses are used for the estimate of the average distance traveled by the domain wall.

compared to $293 \mathrm{~K}$. This result excludes a purely thermal activation of the domain wall displacements by the transient temperature rise after excitation by the laser pulse. In such a case, the difference in the transient temperature would be the same as the difference in base temperature, namely $40 \mathrm{~K}$. Assuming that the temperature rise by the laser pulse is proportional to the number of photons per pulse, i.e., to the fluence, the two curves in Fig. 5 should be horizontally offset by a fixed amount. This difference in fluence between the two curves would then correspond to a transient temperature rise of only $40 \mathrm{~K}$. Apart from the fact that the horizontal difference between the two curves is not constant, also the maximum transient temperatures that would result from that are far too

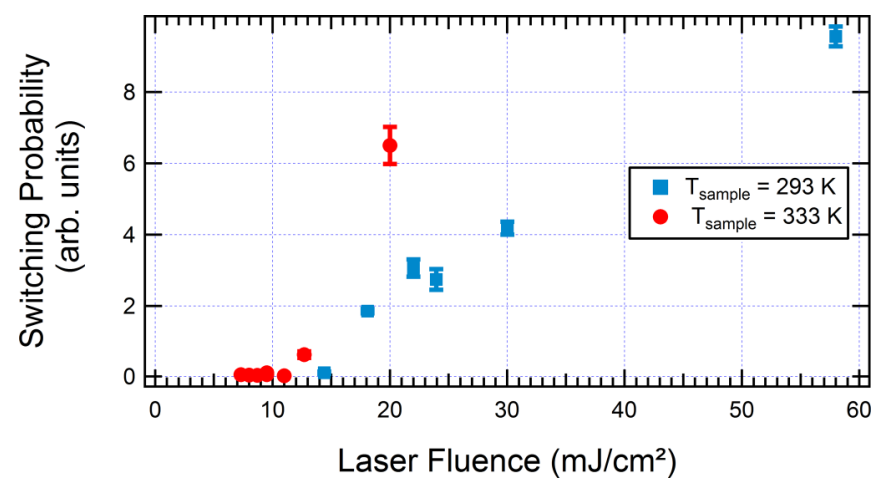

FIG. 5. Switching probability as a function of laser fluence for a sample base temperature of $293 \mathrm{~K}$ (squares) and $333 \mathrm{~K}$ (circles). The error bars are the standard deviation from the statistical analysis. low. It is not possible to describe the observed domain wall displacements by thermal activation with a temperature rise of only some tens of $\mathrm{K}$ for a few nanoseconds when the domains are stable on the order of several minutes at $333 \mathrm{~K}$. The observed laser-pulse-triggered domain wall movement hence needs an additional, more complicated mechanism for explanation. The distance traveled by the domain walls after one pulse is some hundreds of nanometers. Assuming realistic domain wall speeds, which should be clearly below $1000 \mathrm{~m} \mathrm{~s}^{-1}$, the time needed for these distances is at least $1 \mathrm{~ns}$. This excludes superdiffusive spin currents $[11,13]$ in connection with spin-transfer torque [39-45] as the driving force of the domain wall displacement, because these spin currents persist only about $0.5 \mathrm{ps}$ after the pulse [16]. The time after laser excitation before the optically excited electron system equilibrates with the phononic and magnonic system [46] is also too short, typically less than 1 ps [5], such that an electronic effect of the hot electron gas can also be ruled out. The timescale of the transient temperature rise could be of the right order of magnitude. However, as discussed before, it cannot explain the strong dependence on base temperature. For the same reason, it is also incompatible with a model invoking domain wall inertia to explain the longer time scale of domain wall movements. A possible explanation could be that the energy barrier for thermal activation is lowered by the laser pulse. This could be by a laser-induced depinning of domain walls. Once a domain wall is depinned from a site with a deeper pinning potential, it could then travel by thermal activation over a shallower potential landscape until it reaches another strong pinning site. This could be at relatively low velocities, and hence over longer times, such that the laser fluence is less important and the activation by the base temperature comes into play. If after depinning there is not enough thermal energy, the domain wall would just snap back into the old pinning site. Due to the statistical distribution of pinning potentials, the amount of depinned domain walls would continuously depend on the laser fluence, while the overall switched areas in addition would also depend on the sample temperature. At a higher laser fluence more domain walls are depinned that could be potentially moved after the laser pulse. Such a two-step process could thus explain most of the experimental observations. It is also consistent with the larger average domain wall displacement at the higher base temperature. The observation that domain walls sometimes move back to a previous position [Fig. 1(b) and Ref. [37]] is explained by the presence of strong pinning potentials at these sites. The presence of two close-by domain wall pinning sites leads thus to a kind of "domain breathing" effect when the energy barrier separating the two is overcome by a laser pulse, and to a corresponding toggling behavior of the magnetization in the region between the two pinning sites. For energetically equivalent pinning sites, the probability to find the domain wall in either position would be equal. The small increase in average distance traveled by a domain wall with increasing laser fluence could be due to the distance traveled shortly after the laser pulse, when the sample temperature is still above the base temperature, which seems to be less pronounced at $293 \mathrm{~K}$ sample temperature.

The depinning of domain walls may be either due to thermal excitation during the transient rise of sample temperature or 
to transient spin currents acting on the domain wall via spin torque. A time estimate for domain wall depinning could be based on the domain wall width. Depending on the exact nature of the pinning site, already some distortion of a domain wall might be sufficient for depinning. This could happen at timescales of the order of 10 ps [47]. Laser-pulse-induced changes to the spin structure within domain walls have even been reported on the subpicosecond timescale [16], albeit it is not clear whether such changes would be sufficient for depinning a domain wall. From the time estimate one thus cannot exclude one or the other mechanism for the domain wall depinning.

The locally different probability for domain wall motion, as seen, for example, in Fig. 3, could in principle be due to both different pinning properties of domain walls and locally different absorption properties of the sample. However, since the sample appears quite uniform in PEEM images of the laser pulse and spots in which three-photon photoemission could be observed are rare, we conclude that the local differences in laser-induced domain wall motion are mainly caused by variations of the domain wall pinning potential.

The comparison of magnetic domain images taken at the $\mathrm{Co}$ and $\mathrm{Ni} L_{3}$ edges confirms earlier PEEM studies of this trilayer system [28,48]: Due to the weaker magnetic anisotropy of the out-of-plane-magnetized Ni layer compared to the Co layer, the parallel interlayer coupling leads to a tilt of the Ni magnetization into the Co in-plane magnetization direction [28], which is seen in the Ni domain images as a superimposed imprint of the Co domain pattern. In addition, there is some magnetostatic coupling between domain walls in the Ni layer and the Co layer. Depending of the sense of the magnetization change in $\mathrm{Ni}$ and the direction of the walls there, there is a higher probability to find one direction of magnetization of Co on top of Ni domain walls compared to the other [48], see domain images in the Supplemental Material [37].

The domain structure in the Ni layer, as observed from XMCD PEEM domain images at the $\mathrm{Ni} L_{3}$ edge, does not change at the laser fluences used here, except for the coupling-induced tilt in the direction of the Co magnetization. Only at much higher fluences of about $40 \mathrm{~mJ} \mathrm{~cm}^{-2}$ at a base temperature of $333 \mathrm{~K}$ a similar effect as in the Co layer occurs. This could be due to the lower amount of laser power deposited in the Ni layer, or to details of the domain wall structure of the out-of-plane-magnetized Ni layer. Since due to the higher magnetic anisotropy of the Co layer [28] the influence of the $\mathrm{Ni}$ magnetization on the Co layer is much less than vice versa, we think that the observed effect does not require the particular trilayer structure used in our experiments but is more general and should be present also in other systems.

\section{CONCLUSION}

We have demonstrated that single ultrashort laser pulses, significantly shorter than the time needed for macroscopic domain wall motion, can induce significant depinning and motion of domain walls. The resulting random changes in the domain structure appear qualitatively similar to thermal domain wall fluctuations, which in the quasistatic case leads to thermal melting of magnetic domains [26]. However, from the dependence of the effect on laser fluence and sample base temperature, we can exclude a simple thermal activation. The experimental observations can be explained by a two-step model of laser-induced depinning and successive thermal motion of domain walls. The use of this effect for a controlled movement of domain walls by ultrashort laser pulses, where external magnetic fields are used to define the direction of motion, appears feasible.

\section{ACKNOWLEDGMENTS}

We thank A. A. Uenal for technical support during the beamtime. Funding by the DFG (Ku 1115/11-1) is gratefully acknowledged. Y.A.S. gratefully acknowledges the MoHE and the DAAD for a GERLS scholarship. J.V. thanks PHC "Procope" for a grant (Project No. 28558NE). We thank HZB for the allocation of synchrotron radiation beamtime.
[1] M. H. Kryder, E. C. Gage, T. W. McDaniel, W. A. Challener, R. E. Rottmayer, G. Ju, Y.-T. Hsia, and M. F. Erden, Proc. IEEE 96, 1810 (2008).

[2] W. A. Challener, C. Peng, A. V. Itagi, D. Karns, W. Peng, Y. Peng, X. Yang, X. Zhu, N. J. Gokemeijer, Y. T. Hsia, G. Ju, R. E. Rottmayer, M. A. Seigler, and E. C. Gage, Nat. Photon. 3, 220 (2009).

[3] E. Beaurepaire, J. C. Merle, A. Daunois, and J. Y. Bigot, Phys. Rev. Lett. 76, 4250 (1996).

[4] C. Stamm, T. Kachel, N. Pontius, R. Mitzner, T. Quast, K. Holldack, S. Khan, C. Lupulescu, E. F. Aziz, M. Wietstruk, H. A. Dürr, and W. Eberhardt, Nat. Mater. 6, 740 (2007).

[5] B. Koopmans, G. Malinowski, F. Dalla Longa, D. Steiauf, M. Fähnle, T. Roth, M. Cinchetti, and M. Aeschlimann, Nat. Mater. 9, 259 (2010).

[6] A. V. Kimel, A. Kirilyuk, P. A. Usachev, R. V. Pisarev, A. M. Balbashov, and T. Rasing, Nature (London) 435, 655 (2005).

[7] S. Mangin, M. Gottwald, C. H. Lambert, D. Steil, V. Uhlirr, L. Pang, M. Hehn, S. Alebrand, M. Cinchetti, G. Malinowski,
Y. Fainman, M. Aeschlimann, and E. E. Fullerton, Nat. Mater. 13, 286 (2014).

[8] T. A. Ostler, J. Barker, R. F. L. Evans, R. W. Chantrell, U. Atxitia, O. Chubykalo-Fesenko, S. El Moussaoui, L. Le Guyader, E. Mengotti, L. J. Heyderman, F. Nolting, A. Tsukamoto, A. Itoh, D. Afanasiev, B. A. Ivanov, A. M. Kalashnikova, K. Vahaplar, J. Mentink, A. Kirilyuk, T. Rasing, and A. V. Kimel, Nat. Commun. 3, 666 (2012).

[9] A. R. Khorsand, M. Savoini, A. Kirilyuk, A. V. Kimel, A. Tsukamoto, A. Itoh, and T. Rasing, Phys. Rev. Lett. 108, 127205 (2012).

[10] G. Malinowski, F. Dalla Longa, J. H. H. Rietjens, P. V. Paluskar, R. Huijink, H. J. M. Swagten, and B. Koopmans, Nat. Phys. 4, 855 (2008).

[11] M. Battiato, K. Carva, and P. M. Oppeneer, Phys. Rev. Lett. 105, 027203 (2010).

[12] A. Melnikov, I. Razdolski, T. O. Wehling, E. T. Papaioannou, V. Roddatis, P. Fumagalli, O. Aktsipetrov, A. I. Lichtenstein, and U. Bovensiepen, Phys. Rev. Lett. 107, 076601 (2011). 
[13] D. Rudolf, C. La-O-Vorakiat, M. Battiato, R. Adam, J. M. Shaw, E. Turgut, P. Maldonado, S. Mathias, P. Grychtol, H. T. Nembach, T. J. Silva, M. Aeschlimann, H. C. Kapteyn, M. M. Murnane, C. M. Schneider, and P. M. Oppeneer, Nat. Commun. 3, 1037 (2012).

[14] B. Vodungbo, J. Gautier, G. Lambert, A. B. Sardinha, M. Lozano, S. Sebban, M. Ducousso, W. Boutu, K. Li, B. Tudu, M. Tortarolo, R. Hawaldar, R. Delaunay, V. López-Flores, J. Arabski, C. Boeglin, H. Merdji, P. Zeitoun, and J. Lüning, Nat. Commun. 3, 999 (2012).

[15] C. E. Graves, A. H. Reid, T. Wang, B. Wu, S. de Jong, K. Vahaplar, I. Radu, D. P. Bernstein, M. Messerschmidt, L. Müller, R. Coffee, M. Bionta, S. W. Epp, R. Hartmann, N. Kimmel, G. Hauser, A. Hartmann, P. Holl, H. Gorke, J. H. Mentink, A. Tsukamoto, A. Fognini, J. J. Turner, W. F. Schlotter, D. Rolles, H. Soltau, L. Strüder, Y. Acremann, A. V. Kimel, A. Kirilyuk, T. Rasing, J. Stöhr, A. O. Scherz, and H. A. Dürr, Nat. Mater. 12, 293 (2013).

[16] B. Pfau, S. Schaffert, L. Müller, C. Gutt, A. Al-Shemmary, F. Büttner, R. Delaunay, S. Düsterer, S. Flewett, R. Frömter, J. Geilhufe, E. Guehrs, C. M. Günther, R. Hawaldar, M. Hille, N. Jaouen, A. Kobs, K. Li, J. Mohanty, H. Redlin, W. F. Schlotter, D. Stickler, R. Treusch, B. Vodungbo, M. Kläui, H. P. Oepen, J. Lüning, G. Grübel, and S. Eisebitt, Nat. Commun. 3, 1100 (2012).

[17] S. S. P. Parkin, M. Hayashi, and L. Thomas, Science 320, 190 (2008).

[18] S.-H. Yang, K.-S. Ryu, and S. Parkin, Nat. Nanotechnol. 10, 221 (2015).

[19] J. Hohlfeld, S.-S. Wellershoff, J. Güdde, U. Conrad, V. Jähnke, and E. Matthias, Chem. Phys. 251, 237 (2000).

[20] E. Gamaly, Phys. Rep. 508, 91 (2011).

[21] G. R. Henry, J. Appl. Phys. 42, 3150 (1971).

[22] A. V. Kimel, B. A. Ivanov, R. V. Pisarev, P. A. Usachev, A. Kirilyuk, and T. Rasing, Nat. Phys. 5, 727 (2009).

[23] J. Rhensius, L. Heyne, D. Backes, S. Krzyk, L. J. Heyderman, L. Joly, F. Nolting, and M. Kläui, Phys. Rev. Lett. 104, 067201 (2010).

[24] L. Thomas, R. Moriya, C. Rettner, and S. S. P. Parkin, Science 330, 1810 (2010).

[25] O. Portmann, A. Vaterlaus, and D. Pescia, Phys. Rev. Lett. 96, 047212 (2006).

[26] W. Kuch, K. Fukumoto, J. Wang, F. Nolting, C. Quitmann, and T. Ramsvik, Phys. Rev. B 83, 172406 (2011).

[27] M. Kronseder, M. Buchner, H. G. Bauer, and C. H. Back, Nat. Commun. 4, 2054 (2013).

[28] W. Kuch, X. Gao, and J. Kirschner, Phys. Rev. B 65, 064406 (2002).

[29] E. Bauer, J. Phys.: Condens. Matter 13, 11391 (2001).

[30] J. Stöhr, Y. Wu, B. D. Hermsmeier, M. G. Samant, G. R. Harp, S. Koranda, D. Dunham, and B. P. Tonner, Science 259, 658 (1993).
[31] W. Kuch, Appl. Phys. A 76, 665 (2003).

[32] O. Schmidt, M. Bauer, C. Wiemann, R. Porath, M. Scharte, O. Andreyev, G. Schönhense, and M. Aeschlimann, Appl. Phys. B 74, 223 (2002).

[33] U. Atxitia, O. Chubykalo-Fesenko, N. Kazantseva, D. Hinzke, U. Nowak, and R. W. Chantrell, Appl. Phys. Lett. 91, 232507 (2007).

[34] M. Djordjevic and M. Münzenberg, Phys. Rev. B 75, 012404 (2007).

[35] N. Kazantseva, U. Nowak, R. W. Chantrell, J. Hohlfeld, and A. Rebei, Europhys. Lett. 81, 27004 (2008).

[36] U. Atxitia, O. Chubykalo-Fesenko, J. Walowski, A. Mann, and M. Münzenberg, Phys. Rev. B 81, 174401 (2010).

[37] See Supplemental Material at http://link.aps.org/supplemental/ 10.1103/PhysRevB.94.054414 for element-resolved magnetic domain images, analysis of average domain wall displacements, and a movie of a series of images taken with single laser pulses in between.

[38] C. Won, Y. Z. Wu, J. Choi, W. Kim, A. Scholl, A. Doran, T. Owens, J. Wu, X. F. Jin, H. W. Zhao, and Z. Q. Qiu, Phys. Rev. B 71, 224429 (2005).

[39] J. C. Slonczewski, J. Magn. Magn. Mater. 159, L1 (1996).

[40] E. B. Myers, D. C. Ralph, J. A. Katine, R. N. Louie, and R. A. Buhrman, Science 285, 867 (1999).

[41] I. N. Krivorotov, N. C. Emley, J. C. Sankey, S. I. Kiselev, D. C. Ralph, and R. A. Buhrman, Science 307, 228 (2005).

[42] L. Heyne, J. Rhensius, D. Ilgaz, A. Bisig, U. Rüdiger, M. Kläui, L. Joly, F. Nolting, L. J. Heyderman, J. U. Thiele, and F. Kronast, Phys. Rev. Lett. 105, 187203 (2010).

[43] N. Motzko, B. Burkhardt, N. Richter, R. Reeve, P. Laczkowski, W. Savero Torres, L. Vila, J.-P. Attané, and M. Kläui, Phys. Rev. B 88, 214405 (2013).

[44] A. J. Schellekens, K. C. Kuiper, R. R. J. C. de Wit, and B. Koopmans, Nat. Commun. 5, 4333 (2014).

[45] A. J. Ramsay, P. E. Roy, J. A. Haigh, R. M. Otxoa, A. C. Irvine, T. Janda, R. P. Campion, B. L. Gallagher, and J. Wunderlich, Phys. Rev. Lett. 114, 067202 (2015).

[46] M. I. Kaganov, I. M. Lifshitz, and L. V. Tanatarov, Zh. Eksp. Teor. Fiz. 31, 232 (1957) [Sov. Phys. JETP 4, 173 (1957)].

[47] The order of magnitude of the timescale of $10 \mathrm{ps}$ was estimated as follows: The magnetic anisotropy for $4 \mathrm{ML} \mathrm{Co} / \mathrm{Cu}(001) K=$ $760 \mu \mathrm{eV}$ per atom [49] converts to about $2.7 \times 10^{6} \mathrm{~J} / \mathrm{m}^{3}$. Using the bulk value $A=13 \times 10^{-12} \mathrm{~J} / \mathrm{m}$ as exchange constant for Co, this yields $\sqrt{A / K}=2 \mathrm{~nm}$. With a domain wall speed of $200 \mathrm{~m} / \mathrm{s}$, a time of $10 \mathrm{ps}$ is obtained.

[48] W. Kuch, L. I. Chelaru, K. Fukumoto, F. Porrati, F. Offi, M. Kotsugi, and J. Kirschner, Phys. Rev. B 67, 214403 (2003).

[49] M. Kowalewski, C. M. Schneider, and B. Heinrich, Phys. Rev. B 47, 8748 (1993). 